Document downloaded from:

http://hdl.handle.net/10251/122278

This paper must be cited as:

Guardiola, C.; Pla Moreno, B.; Bares-Moreno, P.; Barbier, ARS. (2018). An analysis of the in-cylinder pressure resonance excitation in internal combustion engines. Applied Energy. 228:1272-1279. https://doi.org/10.1016/j.apenergy.2018.06.157

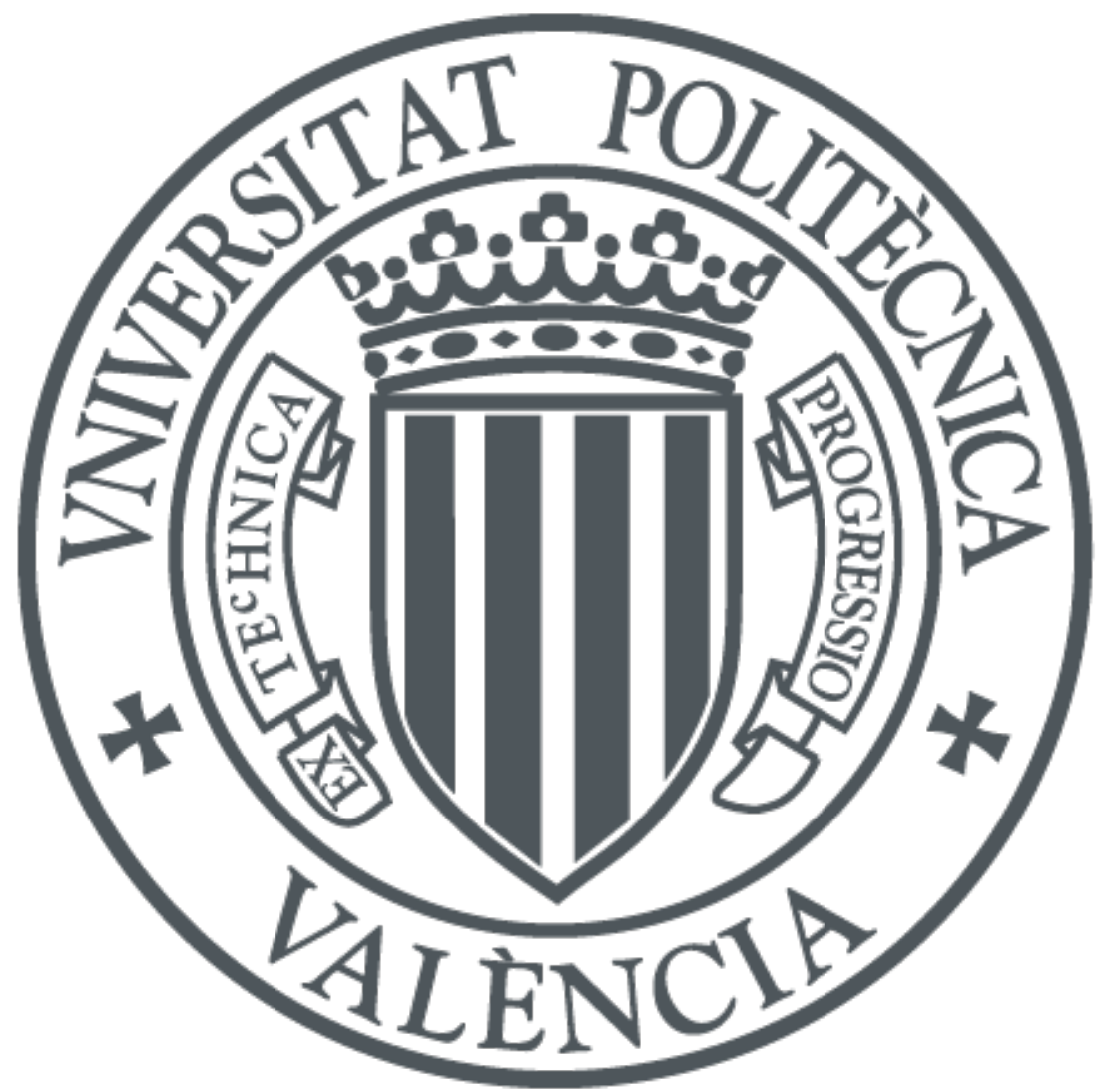

The final publication is available at

http://doi.org/10.1016/j.apenergy.2018.06.157

Copyright Elsevier

Additional Information 


\title{
An analysis of the in-cylinder pressure resonance excitation in internal combustion engines
}

\author{
C. Guardiola B. Pla P. Bares* A. Barbier \\ Universitat politècnica de València
}

\begin{abstract}
This paper analyses the in-cylinder pressure oscillations in internal combustion engines, which are initiated by combustion and resonate during the expansion stroke. A specific mathematical tool, which takes into account the resonant frequency theory, has been developed to determine the resonance intensity evolution from the in-cylinder pressure signal.

Two engines, a conventional spark-ignited (SI) engine modified to perform homogeneous charge compression ignition (HCCI) combustion, and a conventional compression ignited (CI) engine, were used to analyse the resonance excitation in various combustion modes at different operating conditions.

The new transformation has been used to characterize resonance in various combustion modes and a previous method developed by the authors to estimate the trapped mass was fed by such knowledge to improve its robustness and accuracy.
\end{abstract}

Key words: Knock, internal combustion engines, Fourier transform, resonance, in-cylinder pressure

\section{Introduction}

In-cylinder pressure oscillations in internal combustion engines are excited by local pressure gradients created by combustion, and resonate in the cylinder combustion chamber during the expansion stroke.

* Corresponding author:
Email address: pabamo@mot.upv.es (P. Bares). 
The resonance frequency evolution was firstly characterized by Draper in 1938 by analytically solving the wave equation with cylindrical contour conditions [1]. Draper stated that the frequency of the resonant modes was proportional to the speed of sound. In the last decade, as new tools for time-frequency analysis were being developed, several authors have demonstrated the relation between the speed of sound and the frequency of the resonant modes found by Draper,.e.g. Scholl et al. with spectrograms [2], Samimy et al. with the Wigner distribution in [3], or Stanković et al. with the S-method [4]. In engines with bowl, such as compression ignited (CI) engines, the relation between the speed of sound and the resonance frequency may vary from the cylindrical acoustical response, as proved by Torregrosa et al. and Broatch et al. by numerical analysis and experimental results $[5,6]$.

Pressure oscillations caused by resonance are a non-desired consequence of combustion which must be controlled for a safe and efficient operation:

- In spark ignited (SI) engines the main limiting factor at high load is the knock phenomenon, which consists on the autoignition of the end gas, before the flame front reaches all the combustion chamber.The rapid auto-ignition of the end gas heavily excites resonance and its vibration reduces the combustion efficiency and can damage the engine. $\mathrm{Xu}$ et al. demonstrate by numerical studies that the convergence of radial and axial modes in knocking conditions are one of the main causes of engine damage [7]. More detailed reviews of knock can be found in $[8,9]$.

- In CI engines, one of the main concerns for the vehicle commercialization is its associated engine noise, which is strongly affected by the pressure oscillations caused by combustion. Some authors, such as Torregrosa et al. [10] and Payri et al. [11] have characterized the engine noise by a pressure decomposition between pseudo-motored, combustion and resonance to analyse each effect separately, highlighting the relevance of resonance on the final noise level.

- Regarding new combustion modes, which are based on an homogeneous charge compression ignition (HCCI) to perform a high efficient low temperature combustion with low NOx and PM emissions (a review can be found in [12]), the rapid autoignition of the mixture heavily excites resonance and its intensity control is one of the key issues of its future implementation. Commonly, the ringing intesity index developed by J.A. Eng in [13] is used, and a value under $5 \mathrm{MW} / \mathrm{m}^{2}$ is recommended.

Most of resonance related studies are oriented to characterize resonance evolution in order to implement combustion strategies for avoiding knocking conditions (in SI) or reducing resonance excitation (in CI and HCCI modes). 
The main source of resonance excitation in SI engines, knock, is produced at the end of the normal combustion event when the end gas reaches sufficient energy to auto-ignite. D'Adamo et al. found the highest knock probability area by flame visualization, in a laboratory GDI engine with optical access, at the exhaust side of the combustion chamber. This is consistent with the numerical simulations (RANS) performed, which showed that this region suffers of lean and hot end-gases, promoting knock onset [14]. However, although the autoignition of the end gas could be predicted with physic models, such as Livengood and Wu correlation [15, 16] or black box models [17], knock is considered a random phenomenon as unpredicted temperature hot spots or oil deposits may trigger the phenomenon. Pan et al. in [18] analysed the influence of these temperature hot-spots in the knock appearance by performing large eddy simulations (LES) analysis. Several works have been focused on studying the effect of fuel blends in the knock occurrence, such is the case of Wei et al. and Liu et al. with n-butanol gasoline blends [19, 20].

Regarding CI combustion, Kyrtatos et al., in [21, 22], determined that the resonance excitation in a conventional CI combustion is mainly caused by the first pre-mixed ignition, and not the later diffusive combustion. They made a study based on a single injection strategy by varying the operating conditions, and they shown a relation between the time delay (and hence the amount of fuel burnt in premixed conditions) and the intensity of the pressure oscillations. However, current CI engines, use multiple pilot injections to reduce the in-cylinder pressure oscillations [23]. The main goal of the pilot injection is to increase the temperature of the combustion chamber through a first short combustion in order to lower the autoignition delay of the second main injection, and thus the pre-mixed fuel burned. Zhang et al. analysed experimental data at various operating conditions with a two-injection strategy, concluding that an important part of the resonance energy is due to the first pilot combustion [24], and that the phasing and quantity of the main injection may importantly affect the intensity of the pressure oscillations [25].

Several authors analysed HCCI combustion and the consequent pressure oscillations, such as in [26] through fuel tracer LIF and chemiluminescence imaging, in $[27,28]$ by image-intensified high-speed video camera speed, and in [29] through large eddy simulations (LES). These studies determine that the autoignition of the mixture starts at some given local points characterized by in-cylinder inhomogeneities, which explains the cycle-to-cycle variability of the pressure oscillation intensity. Kirsten, M. et al., in [30], differentiated the pressure oscillation caused by an HCCI combustion, named as ringing, with the autoignition of the end gas at the end of the combustion which is known as knock. Li et al. used thermodynamic models [31] and Bahri et al. neural networks [32]combined with experimental data to analyse the ringing 
intensity: both concluded that shortest combustion duration (from CA10 to CA50) are associated with higher ringing intensities. Rahnanma et al. suggested using nitrogen for reducing the reactivity of the mixture and hence maintaining ringing intensities under desirable limits [33], while Ahmadi et al. alerted from knock occurrences when adding hydrogen to methane-diesel reactivity controlled compression ignition (RCCI) engines [34].

The authors have recently published a method for deriving the speed of sound by analysing the frequency of the pressure oscillations and obtaining the trapped mass from the ideal gas law [35, 36]. A direct transformation was also developed by combining the resonance theory with the Fourier transform. The new transformation avoids the computational burden of time-frequency analysis and directly obtains the optimal trapped mass which characterizes the pressure oscillations [37]. The method does not require any calibration for pent-roof combustion chambers while the acoustical response of piston-inbowl combustion chambers can be characterized with experimental data or by performing a numerical analysis of the combustion chamber by finite element method (FEM), as demonstrated in [38]. The measurement of trapped mass obtained from resonance has a cycle-to-cycle time response and does not rely in mass flow sensors, which might be used for improving the accuracy and the transient response of some models, such as proposed in [39] for $\mathrm{NO}_{x}$ modelling , in [40] for exhaust temperature estimation, in [41] for residual gases modelling, or in [42] for knock prediction.

This paper analyses in-cylinder pressure data from two engines in order to characterize the resonance excitation at various combustion modes. Current studies of resonance lack from an appropriate tool for analysing the pressure oscillations that resonate along the combustion chamber. Time-frequency analysis, such as Wigner distribution or STFT, is based on identifying constant frequency harmonics during the piston stroke. However, the frequency of resonance evolves during the piston stroke as a function of the combustion chamber properties. The present paper proposes a compact transformation that combines resonance theory with signal analysis for an adequate analysis of the resonance intensity along the piston stroke. The new mathematical tool can be used to design combustion control strategies to reduce the resonance excitation, to detect knock, or to identify the optimal range to analyse the pressure oscillations for a trapped mass estimation.

The paper is organized as follows: next section describes the two engines used and the tests performed. Section three presents the transformation proposed. Section four aims to characterize each combustion mode and its resonance ex- 
citation by using some of the tests recorded for illustration purposes. Section five illustrates the potential of such knowledge for trapped mass determination, and finally, section 6 collects the main conclusions of the work.

\section{Experimental setup}

Engine A A conventional light-duty spark-ignited engine was modified to enable multi-mode combustion, namely SI, CAI, and SACI. The original engine already dispose a double overhead camshaft (DOHC) with a phasing authority of 50 degrees of crank angle degrees in the VVT system of each valve, which was used to perform NVO strategies. Negative valve overlap (NVO) is used to control the internal residual gases for providing sufficient energy for autoignition $[43,44]$ in CAI combustion, while in spark assisted compression ignited (SACI) mode the flame propagation created by the sparks is used to control the autoignition of the end-gas [45, 46].

A custom piston and head machining allowed to increase the compression ratio from 9.2 to 11.25. A high pressure cooled EGR system was connected downstream of a $58 \mathrm{~mm}$ throttle body. These two elements, the throttle and the EGR, were moved further upstream to ensure a proper mixing. A turbocharger, controlled with a waste-gate valve, was also included in the system layout to provide sufficient intake boost.

The tests conducted in engine A can be divided in two parts: a first test campaign, where the residual mass was controlled by NVO strategies to perform CAI and SACI combustion concepts, and a second one, where conventional SI combustion was used in eight different configurations settings. In all the tests, information from 300 cycles was recorded and the engine speed was maintained at $2000 \mathrm{rpm}$.

Figure 1 gives specific information of each control input for the aforementioned tests.

Engine B Engines B is a four-stroke CI engine with 1.5 litres of total displacement, equiped with common-rail injection system, a high-pressure EGR loop and a single-stage turbocharger. 


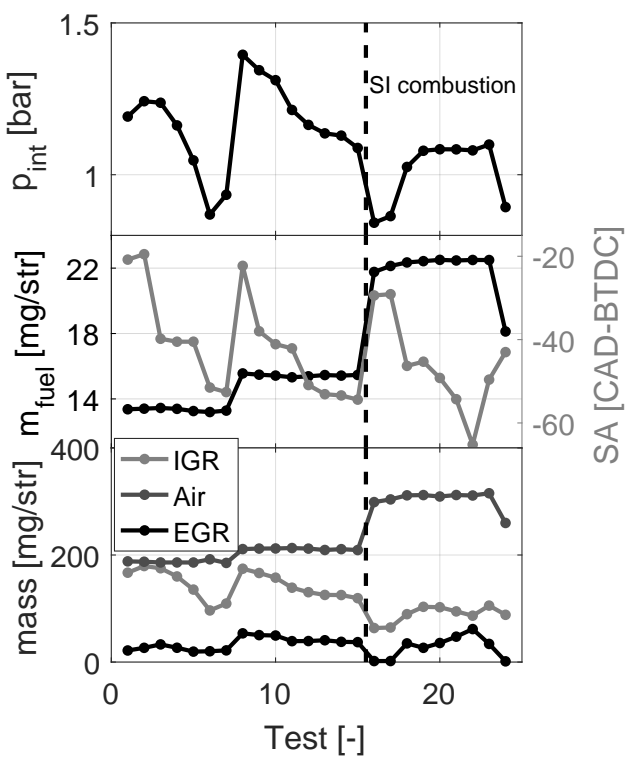

Fig. 1. Intake pressure (top), injection settings (center), and mass components (bottom) of the tests tested in Engine A

A starting dataset of points (with 39 OP), which covers all the operating range, was extended by performing variations over the SOI of the main injection, over the SOI of both injections, namely pilot and main, over the EGR, over the intake pressure, and over the rail pressure. Finally, a total of 342 points were collected. At each test, data from 100 cycles was recorded by starting the measurement when operating conditions were stabilized.

Figure 2 plots the 342 points together, starting map and variations, and points out the clusters where the variations have been performed.

\section{Methodology}

The frequency evolution of the in-cylinder pressure resonance in a cylindrical combustion chamber was derived by C.S. Draper in 1938 [1]. Concluding that the resonance frequency is proportional to the speed of sound, such as:

$$
f_{i, j}=a \sqrt{\frac{B_{i, j}^{2}}{(\pi D)^{2}}+\frac{g^{2}}{(2 h)^{2}}}=\frac{a B_{i, j}}{\pi D}
$$

where the axial modes $g$ are neglected near the TDC because the height is too low $(h<<), D$ is the bore of the cylinder, $a$ the speed of sound, and $B_{i, j}$ are 


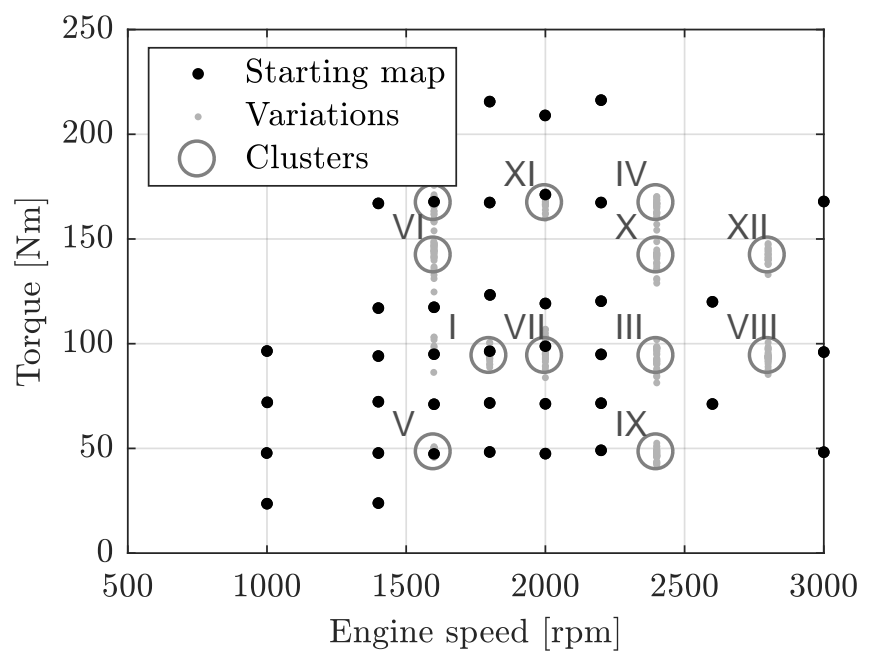

Fig. 2. Steady tests performed in Engine B: Basic operating map is shown as black dots, variations over SOI, EGR, intake pressure, and rail pressure are shown as small grey dots, and clusters are marked and enumerated by big grey cicles

the Bessel constants related with the radial modes.

The speed of sound can be obtained by measuring the trapped mass $m$, the incylinder pressure $p$, and estimating the instantaneous volume of the chamber $V$.

$$
a=\sqrt{\gamma p V / m}
$$

Figure 3, top plot, shows the in-pressure oscillation of a cycle in Engine A at $2000 \mathrm{rpm}$ and low load in a HCCI combustion. The evolution of the amplitude of the harmonics has been computed through a short time Fourier transform (STFT) in the medium plot, and through a Wigner distribution (WD) in the bottom plot. The frequency evolution derived from the cylindrical theory, by (1), is plotted with a continuous black line, and three windows are highlighted with vertical lines.

In [37] the authors suggested a direct transformation by considering harmonics characterized by virtual trapped masses, and not mathematical tools based on constant frequency harmonics, such as the STFT or the WD. The discrete form of the new transformation suggested is defined by:

$$
S(m)=\sum_{\alpha=\alpha_{2}}^{\alpha=\alpha_{1}} w\left(\alpha-\alpha_{1}\right) p_{h p}(\alpha) e^{-j 2 \pi \sum_{\phi=0}^{\phi=\alpha} T(\phi) \frac{\mu(\phi) B_{1,0} \sqrt{\gamma(\phi) p(\phi) V(\phi)}}{\pi D \sqrt{m}}} T(\alpha)
$$

where $\alpha_{1}$ and $\alpha_{2}$ is the interval where the resonance analysis is performed, $w$ is a window function of $\alpha_{2}-\alpha_{1}$ length, and $T(\alpha)$ is the instantaneous sampling rate, which is constant only in time-based in-cylinder pressure acquisition or 


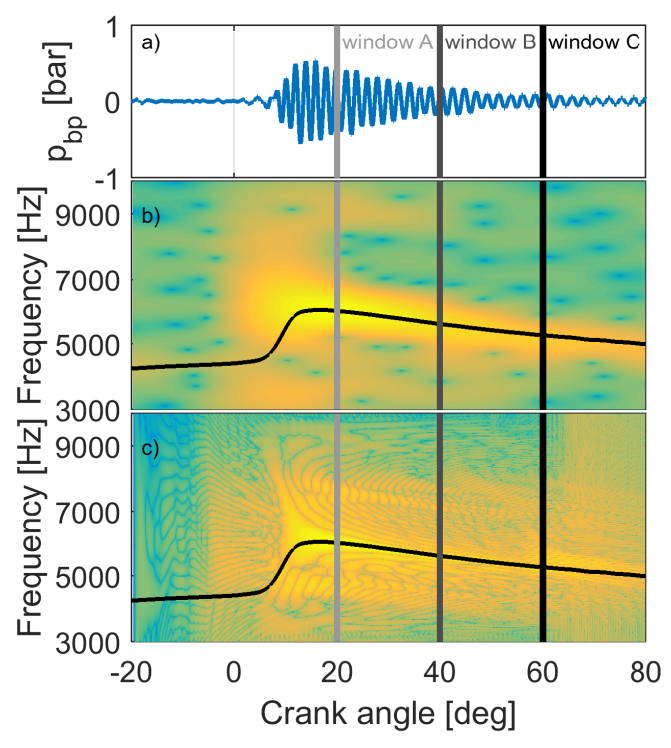

Fig. 3. In-cylinder pressure signal after applying a band-pass filter between $3 \mathrm{kHz}$ and $10 \mathrm{kHz}$ (top), STFT (center) and WD (bottom) of a cycle in Engine A at 2000 rpm and low load in a HCCI combustion

if the instantaneous engine speed fluctuations are negligible.

For a proper analysis the in-cylinder pressure signal must be filtered with a high-pass filter $p_{h p}$ in order to remove the low-frequency content (below the frequency of the first resonant mode), as the low-frequency content has high amplitudes associated and may disturb the final result.

Figure 4 shows the effect of using various windows size and locations: windows A, B and C, are Blackman-Harris of 20 CAD length, centred at 20, 40 and 60 CAD-ATDC, respectively, such as marked in figure 3 , while window $2 x$ makes use of a Blackman-Harris window of $40 \mathrm{CAD}$ centred at $40 \mathrm{CAD}-\mathrm{ATDC}$. The measured trapped mass has been represented by a red vertical line.

Note the effect of the window on the final transformation: a wider window has more resonance information and hence, can be more concise at the trapped mass estimation, while a smaller window will have less computational burden associated. The amplitude of the transformation is higher when the window location approaches the combustion (the source of the excitation), but regardless of the window location the value of the trapped mass remains almost constant.

The present work proposes a modification of the transform presented in (3) to analyse the excitation of the first resonant mode. The method consists in 


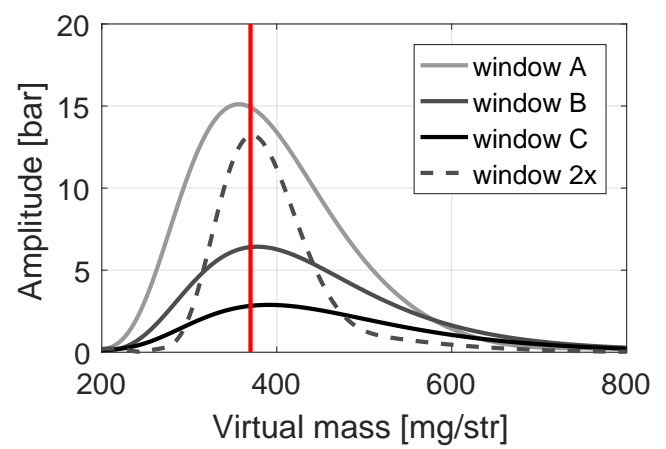

Fig. 4. Direct transform applied to the cycle shown in Figure 3 with various windows size and lengths

using an auxiliary method to estimate the trapped mass, such as sensors or the speed density method, and compute the transformation over various window at each crank angle location, such as:

$$
I_{r}\left(\alpha_{1}\right)=\sum_{\alpha=\alpha_{2}}^{\alpha=\alpha_{1}} w\left(\alpha-\alpha_{1}\right) p_{h p}(\alpha) e^{-j 2 \pi \sum_{\phi=0}^{\phi=\alpha} T(\phi) \frac{\mu(\phi) B_{1,0} \sqrt{\gamma(\phi) p(\phi) V(\phi)}}{\pi D \sqrt{m}}} T(\alpha)
$$

This new parametrization offers, not only an indicator of the first mode resonance excitation, but also where the mode has been excited. Figure 5 shows the new resonance index proposed applied to the cycle shown in Figure 3 by using a Blackman-Harris window of $20 \mathrm{CAD}$ and $\alpha_{1}$ is varied between -20 and 80 CAD-ATDC. Note that resonance is heavily excited when combustion starts and slowly damped during the expansion stroke.

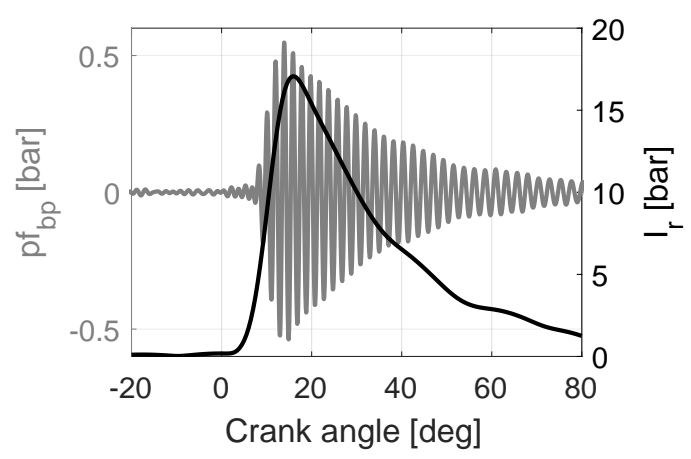

Fig. 5. New resonance index proposed applied to the in-cylinder pressure cycle shown in Figure 3

Next section is devoted to show the potential of such transformation to characterize the resonance evolution in various combustion modes. 


\section{Resonance characterization}

SI combustion Figure 6 shows 30 cycles of conventional SI combustion in engine A at medium load (7 bar of BMEP) and $2000 \mathrm{rpm}$. The top plot shows the in-cylinder pressure evolution low-pass filtered below $4.5 \mathrm{kHz}$, the medium plot shows the heat release rate, and the bottom plot shows the resonance excitation evolution computed by (4).

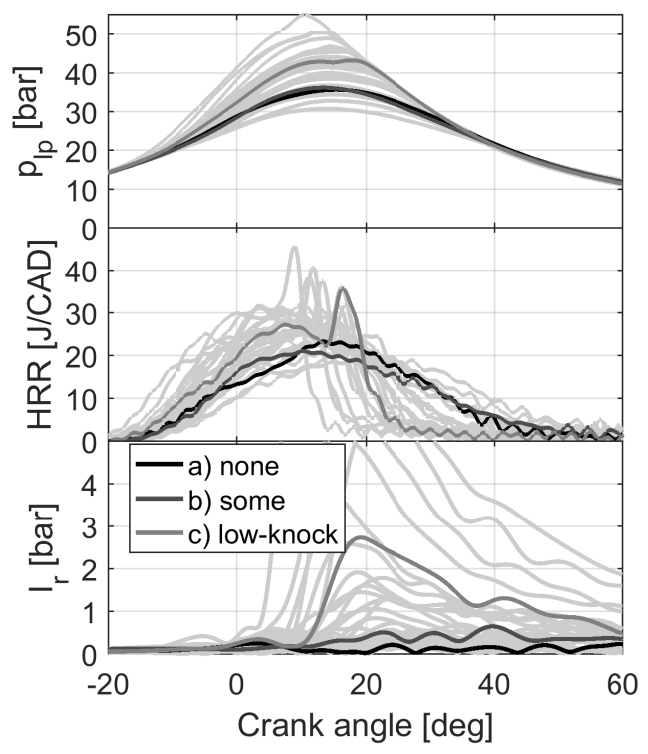

Fig. 6. Low pass filtered pressure signal (top), heat release rate (center) and resonance excitation index (bottom) in 30 cycles of SI combustion at medium load and $2000 \mathrm{rpm}$

Figure 6 shows the first 30 cycles of a wider set of 300 cycles, recorded at steady conditions (point 19). From all the set, only 2 cycles can be considered an actual knock (MAPO $\geq 0.5$ bar), the average of the CA50 is 10.8 CADATDC and its standard deviation 3.9 CAD. Three cycles have been highlighted with a different grey scale in order to illustrate the three types of resonance excitation founded. The in-cylinder pressure signal of these cycles has been filtered with a high-pass filter (above $4.5 \mathrm{kHz}$ ) and it is plotted in Figure 7.

These three cycles represent the three types of resonance excitation found in a SI combustion:

- The first case (cycle a) is an example of some cycles where no resonance exist, i.e. the flame is smoothly propagated till it reaches the end of the combustion chamber without exciting the first mode of the in-cylinder pressure resonance. 


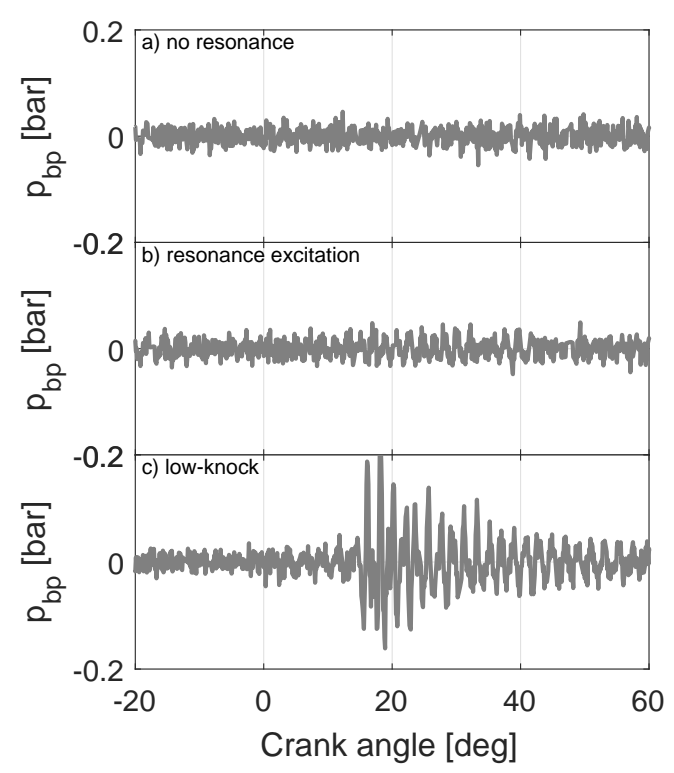

Fig. 7. High-pass filtered pressure signal of the three cycles highlighted in Figure 6

- Many cycles in SI combustion exhibit a smooth pressure evolution but slightly excite the in-cylinder pressure resonance during the flame propagation, such as in cycle b. The resonance excitation in this cycle seems to be exited at various locations during the flame propagation and maintains always a low excitation.

- And finally, in cycles were combustion is initiated far before the TDC, resonance is heavily excited by the autoignition of the end gas. In this case the resonance is highly excited in a concrete location and is damped during the expansion stroke.

SACI-CAI combustion Figure 8 shows the pressure signal (top), heat release rate (center) and resonance evolution (bottom) of 30 cycles of SACI combustion at $2000 \mathrm{rpm}$ and low load, where a cycle has been highlighted in black to illustrate a typical SACI combustion

The resonance excitation in a SACI combustion is always caused by the main combustion, which consists on the autoignition of the end gas, i.e. similar to the third case analysed of the SI combustion. Resonance is excited near the maximum heat release rate and it is damped during the expansion stroke. Because the combustion is triggered by the flame front, which is initiated at the spark, SACI combustion has a cycle-to-cycle variability similar to SI engines, i.e. in this test the average CA50 is 9.8 CAD-ATDC and its standard deviation at the 300 cycles is $3.4 \mathrm{CAD}$.

Figure 9 shows the histogram and a normal distribution fit of the location of 


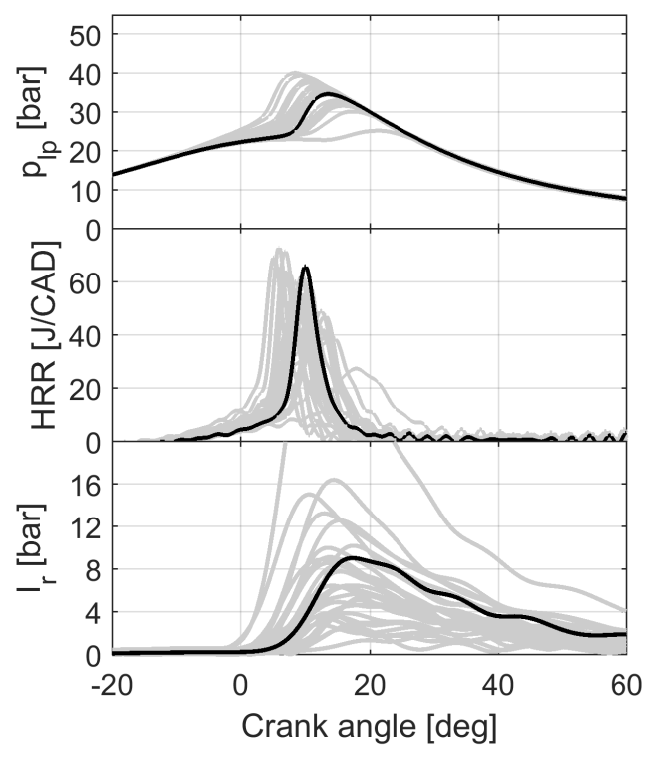

Fig. 8. Low pass filtered pressure signal (top), heat release rate (center) and resonance excitation index (bottom) in 30 cycles of SACI combustion at low load and $2000 \mathrm{rpm}$.

the CA50, the maximum in-cylinder pressure, and the maximum resonance of 300 cycles in SI conventional combustion and in SACI combustion modes. Note that in a SACI combustion the resonance has a predictable behaviour, but in many cycles of SI combustion the resonance is excited during the flame propagation and cannot be predicted so easily.

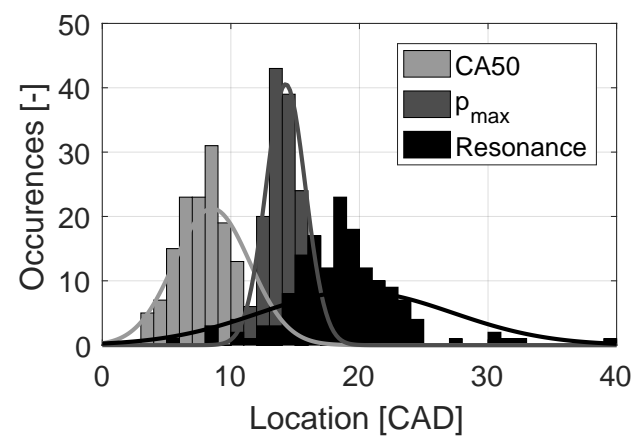

(a) SI

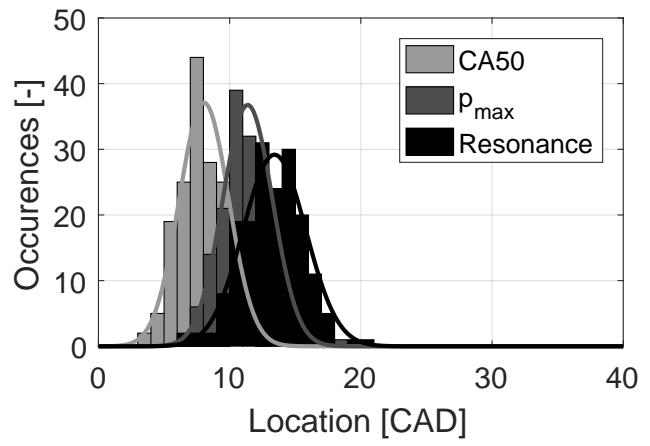

(b) SACI

Fig. 9. Locations of the CA50, the maximum in-cylinder pressure, and the maximum excitation of the resonance, in 300 cycles working at $2000 \mathrm{rpm}$ in Engine A

Figure 10 shows the pressure signal (top), heat release rate (center) and resonance evolution (bottom) of 30 cycles of CAI combustion at $2000 \mathrm{rpm}$ and low load. The transition from SACI to CAI mode is produced when the incylinder chamber conditions permit the autoignition of the mixture before a flame front is developed from the spark, e.g. when the intake pressure is in- 
creased. As consequence, CAI combustion is independent of the spark advance and is not affected by the characteristics of the flame propagation combustion. In this case, that allows reducing the cycle-to-cycle variability, i.e. the standard deviation of the CA50 is 1.44 CAD which contrasts with 3.4 of the SACI and 3.8 of the SI combustion.

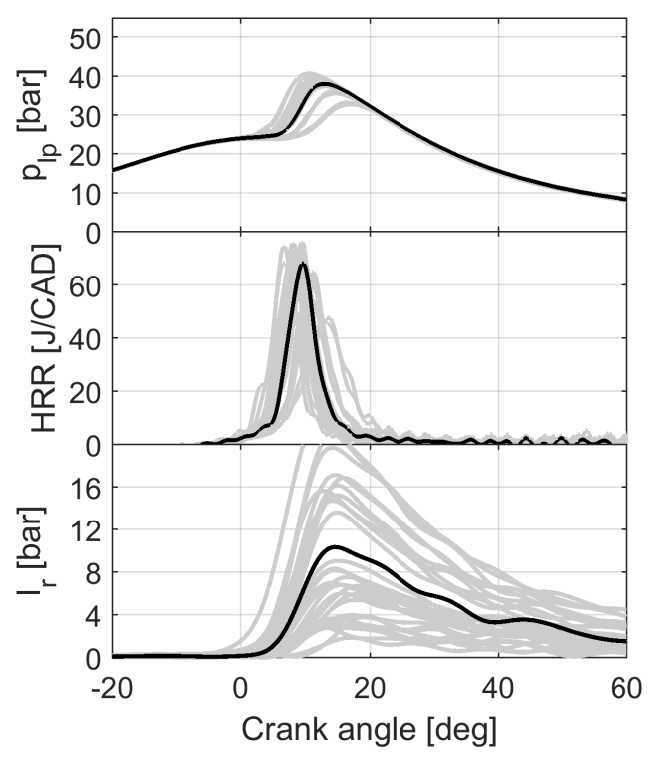

Fig. 10. Low pass filtered pressure signal (top), heat release rate (center) and resonance excitation index (bottom) in 30 cycles of CAI combustion at low load and $2000 \mathrm{rpm}$

CI combustion Figure 11 shows 30 of the 100 cycles recorded at $2400 \mathrm{rpm}$ of engine speed and 8 bar of BMEP, maintaining the same injection settings: $1.4 \mathrm{mg}$ and SOI at $13 \mathrm{CAD}-\mathrm{BTDC}$ for the pilot injection and $19.74 \mathrm{mg}$ and SOI at 5 CAD-ATDC for the main injection. Two cycles are highlighted: cycle $\mathrm{A}$ and cycle $\mathrm{B}$. The former has a higher resonance excitation on the main combustion, while the latter not.

Three conclusions can be pointed out from Figure 11:

- The main contribution to resonance is produced when the first premixed combustion starts, which is consistent with the work performed by Kyrtatos et al. $[21,22]$.

- The resonance excitation of the pilot injection has low cycle-to-cycle variability, while the excitation caused by the main injection substantially varies from one cycle to another. Note that the pilot injection is performed in a mixture of air and EGR, while the main injection is affected by in-cylinder chamber inhomogeneities created by the pilot combustion. 


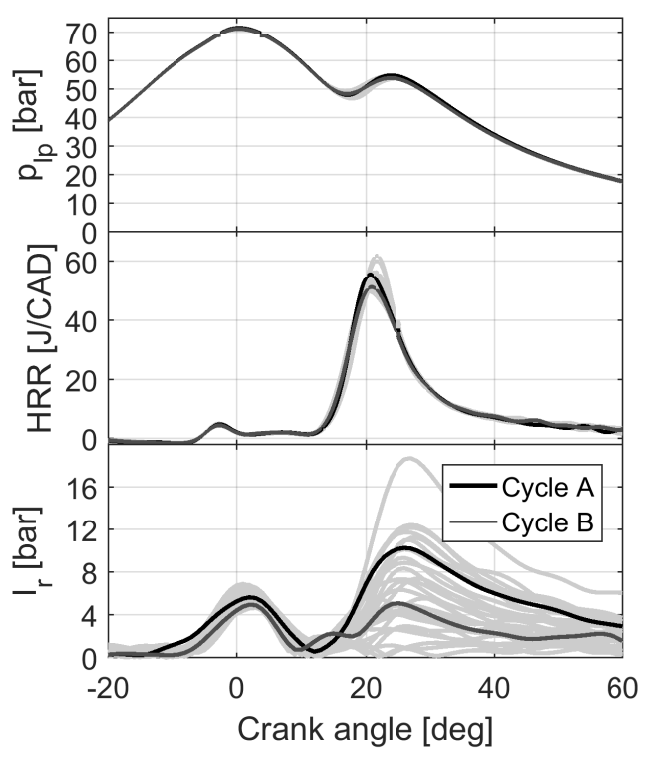

Fig. 11. Low pass filtered pressure signal (top), heat release rate (center) and resonance excitation index (bottom) in 30 cycles of CI combustion with two injections at medium load and $2400 \mathrm{rpm}$

- The resonance excitation of the pilot injection is damped faster than the excitation caused by the main injection. Although the flame propagation might also contribute to the resonance excitation, the damping differences found are also explained because the frequencies near the pilot combustion are substantially higher than those caused by the main combustion.

Figures 12 and 13 show the effect of rail pressure and EGR variations at various operating conditions on the resonance excitation. Note that increasing the rail pressure will always increase the amount of fuel burnt till the autoignition delay is reached, while the EGR smooths the premixed combustion and its resonance excitation.

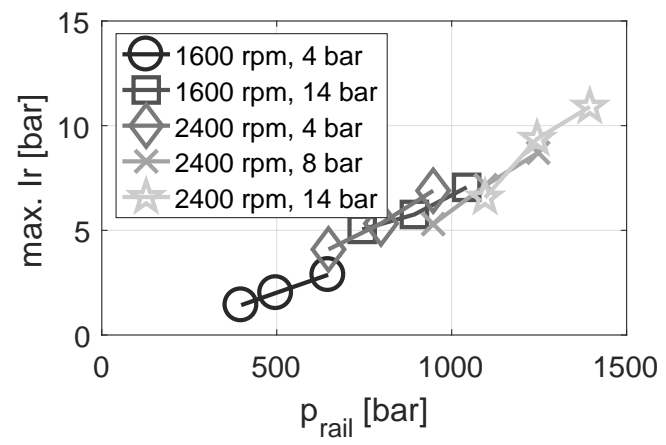

Fig. 12. Average of the maximum resonance index $\left(I_{r}\right)$ of 100 cycles, when varying the rail pressure at various operating conditions 


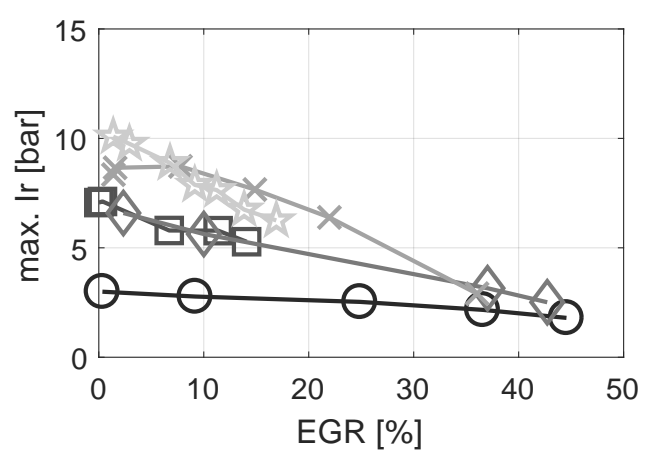

Fig. 13. Average of the maximum resonance index $\left(I_{r}\right)$ of 100 cycles, when varying the EGR at various operating conditions

\section{Application to the trapped mass estimation}

Previous section highlighted the effect of each combustion mode on the resonance evolution. This information could be used to design combustion strategies that might lower the resonance excitation to avoid engine damage and to reduce engine noise. In this section the method will be combined with the trapped mass estimation method ([37]) to use an optimal analysis window that maximises the feasibility for obtaining a good trapped mass estimation from resonance.

The main drawback of the trapped mass estimation method presented in [37] is the requirement of sufficient resonance excitation to analyse the frequency of the in-cylinder pressure oscillations. Figure 14 shows the output of the trapped mass estimation method at a fixed window located at 60 CAD after the TDC in Engine A at test 5. Note that the outliers are characterized by a low resonance excitation.

The analysis range is crucial for an appropriate analysis. In previous works a fixed window was used, herein a moving window is proposed following the scheme shown in Figure 15.

The method proposed is compared with the trapped mass estimation method using two fixed windows: one centred at 20 CAD-ATDC and another at 60 CAD-ATDC, and the accepted cycles have been considered those which have differences with the trapped mass measured by sensors bellow $10 \%$. A blackman-Harris window of $40 \mathrm{CAD}$ was used and the pressure was filtered at $4.5 \mathrm{kHz}$ to separate low from high frequency content. 


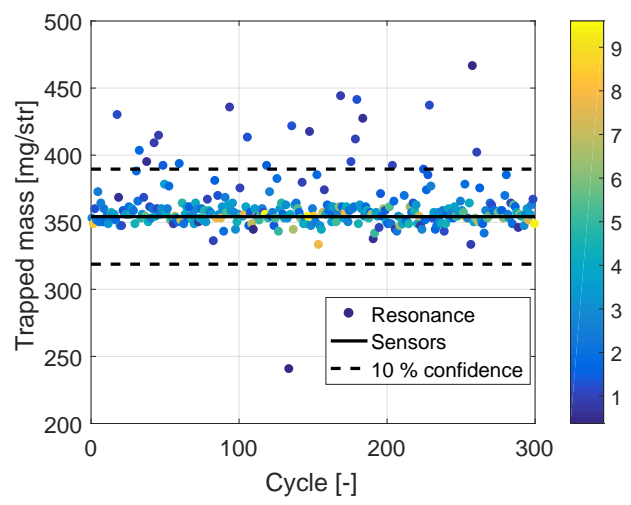

Fig. 14. Trapped mass estimation from resonance in Engine A at test 5 compared with sensors output. Colours indicate the resonance intensity being darker colors less intense oscillations

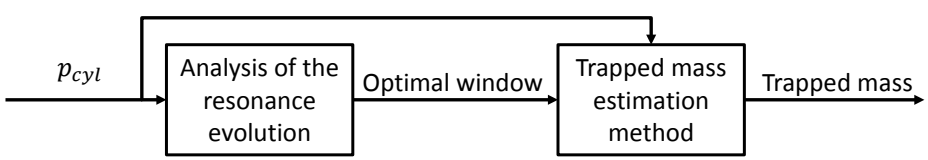

Fig. 15. Scheme of the proposed moving window algorithm to estimate the trapped mass

Engine A Figure 16 shows the percentage of cycles where the resonance was sufficient to estimate the trapped mass in all the tests performed at Engine A. The combustion of the first 15 tests is caused by compression, either autoignited (CAI) or triggered by the flame front of the spark (SACI), while the last 9 tests a conventional SI combustion is developed.

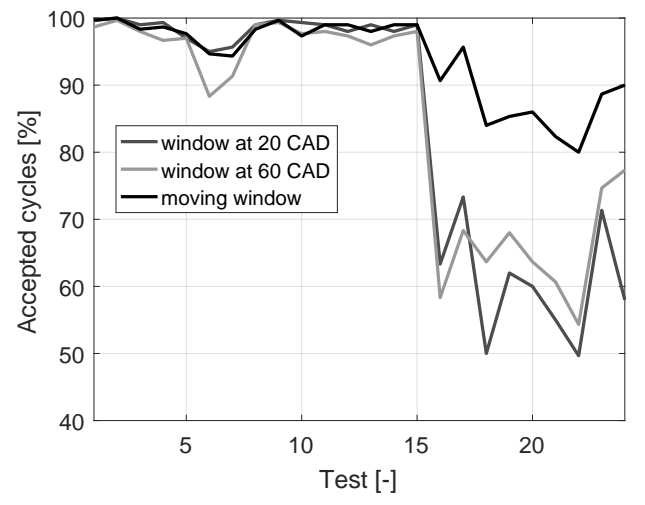

Fig. 16. Percentage of accepted cycles at the resonance estimation for all the tests run in Engine A (SI combustion is identified after test 15) 
It must be noticed that in the first tests, as resonance is heavily excited, all three methods are capable of providing a consistent trapped mass estimation in most of the cycles (only when analysing resonance at $60 \mathrm{CAD}$ some cycles cannot be processed because resonance has been already damped). Nevertheless in the last 9 tests, as resonance is sometimes excited at the end of the combustion because of the end-gas auto-ignition, the method is more efficient at the window located at the end of the stroke (60 CAD-ATDC) than that centred close to the top dead center (20 CAD-ATDC). Regarding the improvements of using a moving window located at the maximum resonance excitation, the method is capable of estimating the trapped mass in around $85 \%$ of the cycles, while a fixed window would provide measurement of only $60 \%$ of the cycles.

Engine B Figure 17 shows the percentage of accepted cycles, when applying the trapped mass estimation method in all the 342 tests of Engine B. Results have been represented by histograms to evidence the number of tests at each range.
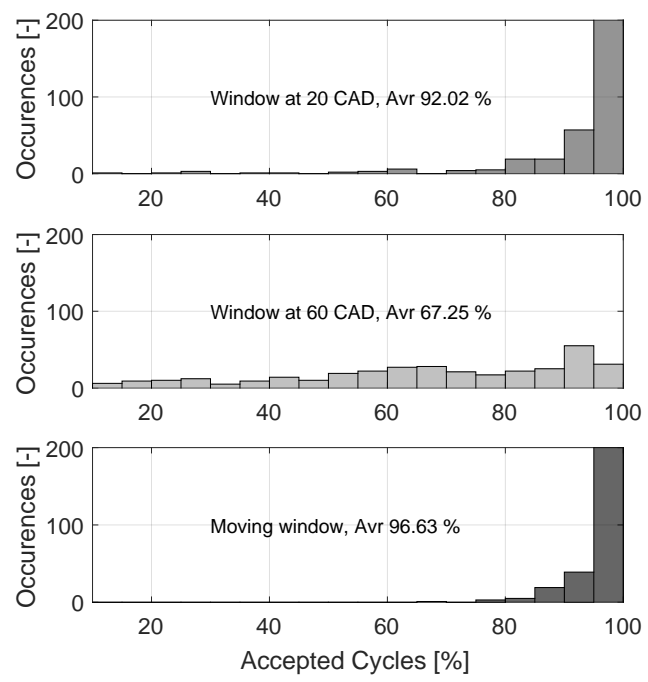

Fig. 17. Percentage of accepted cycles at the resonance estimation for all the tests run in Engine B (conventional CI combustion)

The average of accepted cycles is $92.02 \%$ when using a fixed window at 20 CAD-ATDC, $67.25 \%$ when applying that window at 60 CAD-ATDC, and $96.63 \%$ of the cycles when using a moving window. In this engine, resonance is damped faster, and a window analysis near the TDC is performing better than one at the end of the piston stroke. However, there are some tests that a fixed window does not provide reliable measurements in more than $50 \%$ 
and only a moving window ensures the estimation of the trapped mass in the majority of the cycles in all the tests.

\section{Conclusions}

This paper has presented a new mathematical tool, based on the resonance theory, to analyse the resonance excitation during the piston stroke. The new resonance excitation index has been used in two commercial engines to characterize resonance in different combustion modes.

Results shown that when SI combustion has a smooth flame propagation, resonance is poorly excited, however, small pressure oscillations can be found while combustion is taking place. In many operating conditions, specially at medium and high load conditions where the SA is driven to its limits, lowknocking cycles are detected near the end of combustion, where the maximum of the in-cylinder pressure is located. When the end gas is auto-ignited in lowknocking events, resonance is suddenly excited and resonate during the rest of the expansion stroke.

In new combustion modes, where a mixture of fuel, air and burnt products is auto-ignited, resonance is easily identifiable when combustion takes place. However, the intensity of the pressure oscillation depends on local pressure gradients created when combustion starts, and consequently, resonance excitation may significantly vary from one cycle to the other.

State-of-the-art CI engines use several injection events to reduce soot and noise. Each combustion event is characterized by two phases: a first premixed combustion, when the autoignition delay is reached, and a diffusive flame combustion till the end of the injection event. The resonance excitation is mainly influenced by the amount of fuel burnt in the premixed combustion and the diffusive flame combustion has almost no effect on resonance. When several injections are used, resonance excitation may have a significant cycle-to-cycle variability, as combustion events are affected by inhomogeneities created at previous combustions.

An adequate analysis of the resonance evolution can be used to identify the location where resonance has been excited, the intensity of such oscillation, and the damping of the combustion chamber. This information can be used to 
identify the source of such oscillations in order to design combustion control actions to reduce the engine noise, to study the effect of various combustion chamber designs in the resonance damping or to identify the appropriate crank angle range to analyse resonance.

Here, the analysis of the resonance evolution has been combined with a method for trapped mass estimation that uses resonance to estimate the trapped mass, previously published by the authors. A previous analysis of the resonance evolution, such as suggested in this paper, reduces the number of errors in the trapped mass estimation method. When performing SI combustion the number of faults was reduced from $40 \%$ to a $15 \%$, while in a conventional CI engine the number of errors is reduced from $8 \%$ and $33 \%$ (depending on analysis range chosen) to $3.4 \%$.

\section{References}

[1] C.S. Draper. The physical effects of detonation in a closed cylindrical chamber. Technical report, National Advisory Committee for Aeronautics, 1938.

[2] D. Scholl, C. Davis, S. Russ, and T. Barash. The volume acoustic modes of spark-ignited internal combustion chambers. SAE Technical Papers, 1998.

[3] G. Rizzoni, L. Guzzella, and B.M. Baumann. Unified modeling of hybrid electric vehicle drivetrains. IEEE/ASME Transactions on Mechatronics, 4(3):246-257, 1999.

[4] L. Stanković and J. F. Böhme. Time-frequency analysis of multiple resonances in combustion engine signals. Signal Processing, 79(1):15-28, 1999.

[5] A. J. Torregrosa, A. Broatch, X. Margot, V. Marant, and Y. Beauge. Combustion chamber resonances in direct injection automotive diesel engines: A numerical approach. International Journal of Engine Research, 5(1):83-91, 2004.

[6] A. Broatch, X. Margot, A. Gil, and J. C. Donayre. A cfd approach to diesel engine combustion chamber resonance. SAE Technical Papers, 15, 2007.

[7] H. Xu, A. Yao, C. Yao, and J. Gao. Investigation of energy transformation and damage effect under severe knock of engines. Applied Energy, 203:506-521, 2017.

[8] X. Zhen, Y. Wang, S. Xu, Y. Zhu, C. Tao, T. Xu, and M. Song. The engine knock analysis - an overview. Applied Energy, 92:628-636, 2012. 
[9] Z. Wang, H. Liu, and R. D. Reitz. Knocking combustion in spark-ignition engines. Progress in Energy and Combustion Science, 61:78-112, 2017.

[10] A. J. Torregrosa, A. Broatch, J. Martín, and L. Monelletta. Combustion noise level assessment in direct injection diesel engines by means of in-cylinder pressure components. Measurement Science and Technology, 18(7):2131-2142, 2007.

[11] F. Payri, A. Broatch, X. Margot, and L. Monelletta. Sound quality assessment of diesel combustion noise using in-cylinder pressure components. Measurement Science and Technology, 20(1), 2009.

[12] X. Lu, D. Han, and Z. Huang. Fuel design and management for the control of advanced compression-ignition combustion modes. Progress in Energy and Combustion Science, 37(6):741-783, 2011.

[13] J. A. Eng. Characterization of pressure waves in hcci combustion. SAE Technical Papers, 2002.

[14] A. d'Adamo, S. Breda, S. Fontanesi, A. Irimescu, S. S. Merola, and C. Tornatore. A RANS knock model to predict the statistical occurrence of engine knock. Applied Energy, 191:251-263, 2017.

[15] J. C. Livengood and P. C. Wu. Correlation of autoignition phenomena in internal combustion engines and rapid compression machines. Symposium (International) on Combustion, 5(1):347-356, 1955.

[16] D. Bradley. Autoignitions and detonations in engines and ducts. Philosophical Transactions of the Royal Society A: Mathematical, Physical and Engineering Sciences, 370(1960):689-714, 2012.

[17] G. Panzani, C. Onder, and F. Ostman. Engine knock margin estimation using in-cylinder pressure measurements. IEEE/ASME Transactions on Mechatronics, PP(99), 2016.

[18] J. Pan, H. Wei, G. Shu, M. Pan, D. Feng, and N. Li. Les analysis for autoignition induced abnormal combustion based on a downsized si engine. Applied Energy, 191:183-192, 2017.

[19] H. Wei, D. Feng, M. Pan, J. Pan, X. Rao, and D. Gao. Experimental investigation on the knocking combustion characteristics of n-butanol gasoline blends in a disi engine. Applied Energy, 175:346-355, 2016.

[20] K. Liu, Y. Li, J. Yang, B. Deng, R. Feng, and Y. Huang. Comprehensive study of key operating parameters on combustion characteristics of butanol-gasoline blends in a high speed si engine. Applied Energy, 212:1332, 2018.

[21] P. Kyrtatos, K. Hoyer, P. Obrecht, and K. Boulouchos. Apparent effects of in-cylinder pressure oscillations and cycle-to-cycle variability on heat release rate and soot concentration under long ignition delay conditions in diesel engines. International Journal of Engine Research, 15(3):325-337, 2014.

[22] P. Kyrtatos, C. Brückner, and K. Boulouchos. Cycle-to-cycle variations in diesel engines. Applied Energy, 171:120-132, 2016.

[23] S. Mendez and B. Thirouard. Using multiple injection strategies in diesel combustion: Potential to improve emissions, noise and fuel econ- 
omy trade-off in low cr engines. SAE International Journal of Fuels and Lubricants, 1(1):662-674, 2009.

[24] Q. Zhang, Z. Hao, X. Zheng, and W. Yang. Characteristics and effect factors of pressure oscillation in multi-injection di diesel engine at highload conditions. Applied Energy, 195:52-66, 2017.

[25] Q. Zhang, Z. Hao, X. Zheng, W. Yang, and J. Mao. Mechanism and optimization of fuel injection parameters on combustion noise of di diesel engine. Journal of Central South University, 23(2):379-393, 2016.

[26] A. Hultqvist, M. Christensen, B. Johansson, M. Richter, J. Nygren, J. Hult, and M. Aldjn. The hcci combustion process in a single cycle - speed fuel tracer lif and chemiluminescence imaging. SAE Technical Papers, 2002.

[27] D. Dahl, M. Andersson, and I. Denbratt. The origin of pressure waves in high load hcci combustion: A high-speed video analysis. Combustion Science and Technology, 183(11):1266-1281, 2011.

[28] A. Iijima, M. Tanabe, K. Yoshida, H. Shoji, N. Itoh, A. Terashima, and T. Tojo. Visualization and spectroscopic measurement of knocking combustion accompanied by cylinder pressure oscillations in an hcci engine. SAE International Journal of Engines, 6(4), 2013.

[29] Z. Wang, F. Wang, and S. . Shuai. Study of engine knock in hcci combustion using large eddy simulation and complex chemical kinetics. $S A E$ Technical Papers, 2014-October, 2014.

[30] M. Kirsten, G. Pirker, C. Redtenbacher, A. Wimmer, and F. Chmela. Advanced knock detection for diesel/natural gas engine operation. $S A E$ International Journal of Engines, 9(3), 2016.

[31] Y. Li, M. Jia, Y. Chang, S. L. Kokjohn, and R. D. Reitz. Thermodynamic energy and exergy analysis of three different engine combustion regimes. Applied Energy, 180:849-858, 2016.

[32] B. Bahri, M. Shahbakhti, K. Kannan, and A. A. Aziz. Identification of ringing operation for low temperature combustion engines. Applied Energy, 171:142-152, 2016.

[33] P. Rahnama, A. Paykani, and R. D. Reitz. A numerical study of the effects of using hydrogen, reformer gas and nitrogen on combustion, emissions and load limits of a heavy duty natural gas/diesel rcci engine. Applied Energy, 193:182-198, 2017.

[34] R. Ahmadi and S. M. Hosseini. Numerical investigation on adding/substituting hydrogen in the cdc and rcci combustion in a heavy duty engine. Applied Energy, 213:450-468, 2018.

[35] C. Guardiola, B. Pla, D. Blanco-Rodriguez, and P. Bares. Cycle by cycle trapped mass estimation for diagnosis and control. SAE Int. J. Engines, $7(3), 2014$.

[36] J. M. Luján, C. Guardiola, B. Pla, and P. Bares. Estimation of trapped mass by in-cylinder pressure resonance in HCCI engines. Mechanical Systems and Signal Processing, 2015. Article in Press.

[37] A. Broatch, C. Guardiola, B. Pla, and P. Bares. A direct transform for 
determining the trapped mass on an internal combustion engine based on the in-cylinder pressure resonance phenomenon. Mechanical Systems and Signal Processing, 62:480-489, 2015.

[38] A. Broatch, C. Guardiola, P. Bares, and F.D. Denia. Determination of the resonance response in an engine cylinder with a bowl-in-piston geometry by the finite element method for inferring the trapped mass. International Journal of Engine Research, 2015.

[39] C. C. Guardiola, J. Martín, J. Pla, and P. Bares. Cycle by cycle nox model for diesel engine control. Applied Thermal Engineering, 110:10111020, 2017.

[40] C. Guardiola, P. Olmeda, B. Pla, and P. Bares. In-cylinder pressure based model for exhaust temperature estimation in internal combustion engines. Applied Thermal Engineering, 115:212-220, 2017.

[41] C. Guardiola, V. Triantopoulos, P. Bares, S. Bohac, and A. Stefanopoulou. Simultaneous estimation of intake and residual mass using in-cylinder pressure in an engine with negative valve overlap. IFACPapersOnLine, 49(11):461-468, 2016.

[42] P. Bares, D. Selmanaj, C. Guardiola, and C. Onder. Knock probability estimation through an in-cylinder temperature model with exogenous noise. Mechanical Systems and Signal Processing, 98:756-769, 2018.

[43] J. Hunicz and P. Kordos. An experimental study of fuel injection strategies in CAI gasoline engine. Experimental Thermal and Fluid Science, 35(1):243-252, 2011.

[44] P. Adomeit, A. Sehr, R. Weinowski, K. G. Stapf, D. Seebach, S. Pischinger, K. Hoffmann, D. Abel, F. Fricke, H. Kleeberg, and D. Tomazic. Operation strategies for controlled auto ignition gasoline engines. SAE International Journal of Engines, 2(1):164-172, 2009.

[45] W. Lin, J. Sterniak, and S. V. Bohac. $\mathrm{No}_{x}$ emissions characterization during transient spark assisted compression ignition (SACI) engine operation. In ASME 2013 Internal Combustion Engine Division Fall Technical Conference, ICEF 2013, volume 1, 2013.

[46] L. Manofsky, J. Vavra, D. Assanis, and A. Babajimopoulos. Bridging the gap between HCCI and SI: Spark-assisted compression ignition. SAE Technical Papers, 2011. 